\title{
Molecular recognition of isovanillin crosslinked carrageenan biocomposite for drug delivery application
}

\author{
Fatmawati Adam ${ }^{a, b}$, Mohd Aiman Hamdan ${ }^{a}$, Siti Hana Abu Bakar ${ }^{a}$, Mashitah Mohd Yusoff $f^{c}$, and \\ Rajan Jose $e^{d}$ \\ ${ }^{a}$ Faculty of Chemical and Process Engineering Technology, Universiti Malaysia Pahang, \\ Lebuhraya Tun Razak, Gambang, Kuantan, Pahang, Malaysia; \\ ${ }^{b}$ Centre of Excellence for Advanced Research in Fluid Flow (CARIFF), Universiti Malaysia Pahang, \\ Lebuhraya Tun Razak, Gambang, Kuantan, Pahang, Malaysia; \\ ${ }^{\mathrm{C}}$ Faculty of Industrial Sciences \& Technology, Universiti Malaysia Pahang, Lebuhraya Tun \\ Razak, Gambang, Kuantan, Pahang, Malaysia; \\ ${ }^{d}$ Nanostructured Renewable Energy Materials Laboratory, Faculty of Industrial Sciences and \\ Technology, Universiti Malaysia Pahang, 26300, Kuantan, Malaysia
}

\begin{abstract}
This work aims to evaluate carrageenan as a potential biocomposite for hard capsule in the drug delivery application. The interaction between carrageenan and isovanillin was studied by modeling their molecule electronic surface potential and enthalpy. Simulation results showed that the physical crosslinking is established at two points: hydroxyl (isovanillin)-sulfate (carrageenan) and aldehyde (isovanillin)-hydroxyl (carrageenan) with strong hydrogen bonds (H-bond) lengths of 1.74-1.79 $\AA$. FTIR showed that isovanillin addition increased the intermolecular $\mathrm{H}$-bond up to $57 \%$ as the $\mathrm{OH}$-stretching is shifted from 3376 to $3356 \mathrm{~cm} \_1$. At 3 wt.\% isovanillin concentration, the tensile strength of the biocomposite film increased to $49 \%$ and the hard capsule developed from the formulation solution disintegrated in 9min. Upon contact with water, the $\mathrm{H}$-bonds broke and caused the carrageenan fragments to dissolve in deionized water. The disintegration and mechanical properties of the crosslinked carrageenan biocomposite film made from renewable and low price materials facilitate the application of the film as hard capsules for drug delivery.
\end{abstract}

\section{KEYWORDS}

Biocomposite film; Hard capsule; Hydrogen bonding; Isovanillin; Renewable materials 


\section{FUNDING}

This work was supported by the UMP-Community Technology Solution Platform Fund under Grant UIC160906; Ministry of Science Technology and Innovation via 3 EScience Grant Scheme under Grant UMPRDU 140504; and Postgraduate Research Scheme under Grant PGRS 170377. 